\title{
A Fading Memory Model for Indoor Evacuation - Preliminary Results
}

\author{
$\underline{\text { H. Zhao }}^{\mathrm{a}}$, N. Ronald ${ }^{\mathrm{a}}$ and S. Winter ${ }^{\mathrm{a}}$ \\ ${ }^{a}$ Department of Infrastructure Engineering, The University of Melbourne, Parkville, VIC 3010, Australia \\ Email: haifengz@student.unimelb.edu.au
}

\begin{abstract}
Observations of a process in an environment happen over time and their validity can be ephemeral. Thus, knowledge of the environment may quickly become out of date, which matters especially in safetycritical applications. For example, emergency situations happen after sudden changes to the environment and are continuing to change the environment, while people are trying to evacuate based on their outdated spatial knowledge. This means, the value of time-stamped spatial knowledge fades over time. While historical spatial knowledge can be an accurate mapping of the historical environment, the validity of this knowledge in a changed or changing environment should be considered when used for decision making in the current environment.
\end{abstract}

This paper combines spatial knowledge and the time when the knowledge has been acquired in investigating whether fading memory is beneficial in dynamic environments. Fading memory devalues spatial knowledge with time, trusting more recent explorations more than other ones. The hypothesis of this paper is that fading memory is beneficial for agents decision making in a dynamic environment. The models of fading memory, and route planning based on the age of knowledge have been implemented and tested for indoor evacuation scenarios through agent-based simulation with Repast Simphony. Experiments suggest that this model is potentially a better representation of the dynamic environment in terms of providing evacuation guidance, which also has implications for all kinds of spatial analysis.

Although the test shows that in most cases decision making has benefited from fading memory, there is still some cases where applying fading memory deteriorates the results, which throws up fundamental ethical questions: A service devaluing information that actually still is true may come up with suboptimal (evaluation) information. The reason for this is not the imperfection of fading memory per se, but the simplicity of heuristics applied in the experiments when exemplifying fading memory for testing purpose. In the experiments of this paper, the correctness of the heuristics corresponds to its coincidence with the mechanism of how fire spreads, which is beyond the task in this paper. A sophisticate design of heuristic rules when applying fading memory should improve the performance, and in an ideal situation, guarantee in all cases that applying fading memory leads to better results.

Keywords: Spatial knowledge, dynamic environment, evacuation, routing, agent-based simulation 


\section{INTRODUCTION}

Agents travelling in search for target destinations in a dynamic environment is a common research topic. The agents may be evacuees trying to find their way out of a building in the event of an emergency or service robots moving towards a destination in an indoor environment with roaming kids and movable furniture, robots collaboratively exploring unfamiliar terrain, or vehicles travelling on road networks with crossing pedestrians. The problem is that such environments change all the time. While in (sufficiently) static environments learned and accumulated spatial knowledge may be trustworthy for decision making, in dynamic environments the prior experience of the environment may form out of date knowledge. How to fulfil the wayfinding tasks of agents with potentially outdated knowledge is the research question addressed in this paper.

Reliable shortest path planning in a dynamic environment is to a limited extend possible with a centralised, real-time sensing system that creates situation aware pictures for each agent. However, such a centralised service will in most cases not exist due to lack (or damage) of sensing and communication infrastructure. In this situation traditionally robots fall back to autonomous and exploratory wayfinding operations. Only recently decentralized collaborative wayfinding has been suggested (Richter et al., 2013).

Not much research can be found addressing the reliable shortest path problem from the perspective of a representation of the dynamics in the environment. From an economic perspective, looking at the economic value of data, Krek (2002) has studied the impact of out-of-date data to decision making. In other research the impact of prediction of future states on decision making has been studied; results demonstrated a high sensitivity for the validity of the extrapolation (Wang et al., 2014). The current paper, in contrast, suggests a map with fading memory by adding the temporal dimension to the acquired spatial knowledge, and makes an experiment to test the performance of shortest route planning considering the age of information in memory.

Fading memory devalues spatial knowledge with time, trusting more recent explorations more than other ones. The hypothesis of this paper is that fading memory is beneficial for agents decision making in a dynamic environment. If this hypothesis is true it has implications for all kinds of spatial analysis, but also brings up questions of weighing and of ethics: A service devaluing information that actually still is true may come up with suboptimal (evaluation) information (Freudenburg, 1988).

The rest of this paper is organised as follows. Section 2 presents a short literature review. Section 3 outlines the fading memory model. The implementation of experiments and results are presented in Section 4 and Section 5. The results are discussed in Section 6 and Section 7 will contain some concluding remarks.

\section{LITERATURE REVIEW}

A review for techniques used in indoor human navigation systems can be found in Fallah et al. (2013), where technologies for indoor navigations are compared in four groups: locating the user, planning a path, representing the environment and interacting with the user. Spatial models for context-aware indoor navigation systems are reviewed by Afyouni et al. (2012).

Evacuees can be localised via QR code, Radio frequency identification (RFID) (Rueppel and Stuebbe, 2008), Wireless LANs (WLANs), WiFi, or other location-based services (Lee, 2007). Indoor space is more complex than outdoor space such as road network, and many researchers have worked on this (e.g., Tang and Ren, 2012). Richter et al. (2011) from a cognitive perspective conceptualise indoor space into three hierarchical dimensions. Path planning is to find an optimal route navigating a person from current location to a destination while minimising the travel time (Ahn and Han, 2011), travel distance, or hazard (Kulyukin et al., 2006).

Path planning algorithms use graphs (e.g., Becker et al., 2009) or grids (e.g., Afyouni et al., 2012) to represent the environment. Most of current navigation systems use Dijkstra or A* algorithms. The navigation data can be stored and treated in local database (e.g., Fischer et al., 2008), or a central database (e.g., Amemiya et al., 2004) through wireless connection. Park et al. (2009) notice the changing riskiness in disasters, propose a real-time routing algorithm for rescuers. They use a simple network model to represent the building structure, FDS (Fire Dynamics Simulator) to simulate the smoke spreading, and Dijkstras algorithm to calculate the shortest path with time-weighted links.

Serrão et al. (2012) develop a system integrating GIS information of a building with landmarks detected from a hand-held camera for navigating blind or visually impaired persons in complex buildings. Routes are generated based on the neighbourhood relations of room spaces and are updated in real-time in case that the person fails to follow the assumed-to-walk route. Nguyen et al. (2013) emphasise the a fact that evacuees should follow the boundaries of walls and obstacles in condition of limited visibility because of smoke. They try to develop 
a more realistic simulation of emergency situation by taking into account the smoke diffusion and its effect on the evacuation.

\section{FADING MEMORY}

Imagine a simple evacuation scenario: A certain number of people are lingering in a building, then an event (such as a fire, a gas leakage, a flooding, an earth quake, or a roaming sniper) triggers an alarm, and people start to evacuate. In an evacuation, the first priority is to quickly escape from the unsafe building while minimizing the total risk of harm suffered. This means people take into consideration escape route lengths, but also every additional information available about the location and behaviour of the event in order to assess the risk of alternative routes. While route lengths can be computed from static knowledge of the environment, the dynamics of the event, and its potential to block routes or to expose evacuees to risks of harm, such as smoke inhalation or exposure to high temperature levels, suggests that an update mechanism of the static knowledge is highly desirable.

The indoor space of a building can be represented by a route graph $G=(V, E)$, which consists of a set $V=\left\{v_{1}, v_{2}, \ldots, v_{K}\right\}$ of vertices and a set $E=\left\{e_{1}, e_{2}, \ldots, e_{N}\right\}$ of edges, where $K$ is the total number of vertices and $N$ is the total number of edges. The route graph embedded in $\mathbb{R}^{3}$ draws a map of the initial connectivity of each subspaces of the indoor environment.

In order to provide a generic representation and reasoning model, independent from the type of event, the dynamic state of the edges of a route graph is represented by a generic attribute called state. This attribute shall have three values (Wang et al., 2014): normal, at risk, and blocked. Each of these states corresponds to a weight for shortest path computations. The knowledge for an edge in the route graph is defined as a memory fragment:

Definition 1. A memory fragment is an edge attached with an attribute describing its state and an attribute $t$ describing the specific time when this memory fragment has been acquired or has been last updated:

$$
m=(e, \text { state }, t)
$$

where $e$ denotes one edge of the route graph, state denotes the value of the state attribute associated with that edge, and $t$ denotes the last time this memory fragment was updated.

Definition 2. A fading memory fragment is a memory fragment considered at the current time:

$$
f m=\left(e, \text { state }, t_{c u r}-t\right)
$$

Accordingly:

Definition 3. A static memory is a set of memory fragments acquired by an evacuee prior to an event, denoted by $M_{s}$, and considered valid before the event, $t_{p}<t_{\text {event }}$.

$$
M_{s}=\left\{\left(e, \text { state }, t_{p}\right)_{i}\right\}
$$

where $i \in\{1, \ldots, N\}$, and $t_{p}$ being constant over all fragments and $t_{p} \leq t_{1}, \ldots t_{N}$.

A static memory collects all the knowledge acquired by an evacuee, discarding information of the time when that knowledge has been acquired or was last updated. However, the state of the space as it had been experienced in the past may have changed by dynamic events, thus the reliability of this static knowledge may significantly vary between different memory parts. In comparison, a fading memory fragment reflects not (only) the time when the individual knowledge has been acquired/last updated, but rather how much each memory part is outdated.

Definition 4. A fading memory is a set of fading memory fragments, denoted by $M_{f}$.

$$
M_{f}=\left\{\left(e, \text { state }, t_{c u r}-t\right)_{i}\right\}
$$

where $i \in\{1, \ldots, N\}$ and $t_{\text {cur }}$ is constant over all fragments. A fading memory can be represented by a state table (see Table 1).

In particular, if $t=t_{c u r}$, this fading memory fragment is a real-time memory fragment, which reflects the real-time state of a subspace. 
H. Zhao et al., A Fading Memory Model for Indoor Evacuation...

Table 1. Fading memory state table, at an arbitrary time $t_{c u r}$

\begin{tabular}{|c|c|c|}
\hline Edge & State & Last updated \\
\hline$e_{1}$ & state $_{1}$ & $t_{1}$ \\
\hline$e_{2}$ & state $_{2}$ & $t_{2}$ \\
\hline$\cdots$ & $\cdots$ & $\cdots$ \\
\hline$e_{n}$ & state $_{n}$ & $t_{n}$ \\
\hline
\end{tabular}

Definition 5. A real-time memory consists of fading memory fragments that are valid (sensed) at the current time, denoted by $M_{r}$.

$$
M_{r}=\left\{(e, \text { state }, 0)_{i}\right\}
$$

where $i \in\{1,2, \ldots, N\}$.

For decentralised evacuation, real-time memory is not available.

\section{EXPERIMENT SETTINGS}

The models of fading memory, and route planning based on the age of knowledge have been implemented and tested through agent-based simulation with Repast Simphony. The experiment adopts a five-floor office building as a sample evacuation environment, and generates the route graph of this building with YAMAMOTO (Stahl and Schwartz, 2010). The route graph consists of 1739 edges and 1120 nodes. 150 evacuees are supposed to be placed at predefined locations when the events start, and then walk following the path generated from the route graph at a constant speed $(1.5 \mathrm{~m} / \mathrm{s})$ during the evacuation.

The propagation of the emergency events is simulated through simulating the behaviour of an imaginary sensor graph described by Wang et al. (2014). The real-time situation of the environment is assumed to be monitored with a virtual sensor graph composed of 388 sensors covering every node and edge in the route graph. The emergency event can be represented by the state of sensors. In the simulation sensors update states in each time interval of 10 seconds. For calculation efficiency, each simulation of emergency event only run for 600 seconds, evacuations that exceed this time limitation will be considered as incomplete evacuations and will be disregarded. 2040 different events are simulated, recorded and recalled when comparing evacuation scenarios.

For simplicity, the paper assumes that people have complete knowledge of the connectivity of the subspaces of the environment before the event, and apply two mechanisms of learning here:

- People acquire updated knowledge of the environment on physical encounter with a changed environment.

- People acquire updated knowledge of the environment on encounter with fellow evacuees by exchanging their mutual time-stamped knowledge.

Evacuation routes are generated based on evacuees' memory of the environment, and will be adjusted when their memory of the environment is updated. The objective of routing in this experiment is to evacuate people successfully while minimizing the harm exposure. Considering whether evacuation will adopt fading memory or not, experiment will compare evacuation with two strategies:

- Fading share strategy $(F S)$ : When two people meet they share all their updated knowledge, overriding any outdated information. Routes are generated and adjusted with fading memory relying more on recently updated information, and less so on the older or never contested information.

- Conservative share strategy $(C S)$ : When two people meet they share all their updated knowledge, overriding any outdated information. Routes are generated and adjusted assuming all their knowledge of the environment to be static memory and completely trusting that knowledge without considering the time when the knowledge is updated.

When applying fading memory, both spatial and temporal factors should be considered. The following heuristic rules have been assumed: 
H. Zhao et al., A Fading Memory Model for Indoor Evacuation...

- Temporally, old knowledge tends to be less trustworthy than new knowledge, such that the states of old knowledge are likely to deviate from real-time state more than new knowledge.

- Spatially, knowledge for space close to the source of dynamic events fades more quickly, correspondingly the states of edges that directly neighboured with an edge with a state of blocked will transfer faster than edges adjacent with only at risk edges, and faster than edges linking with only normal edges.

- When the state of an edge is assessed to have deviated so much from current state that a limitation has been exceeded, the state of that edge will be regarded as blocked.

For testing purpose, experiments assume that if the harm that inferred from fading memory is assessed to be three times the value of that for at risk edges, then the knowledge is considered to have deviated too much, so that the state will be regarded as blocked at current time for route planning, which is arbitrary but can be replaced by more sophisticated and preciser mechanisms for problem-specific applications.

\section{RESULTS}

Experiments simulate evacuation with 150 evacuees under 2040 predefined emergency cases with two strategies: evacuating with $F S$ and $C S$. The mean number of successful evacuees for $C S$ is 134.9 , and for $F S$ is 137.6.

Figure 1 classifies the 2040 event cases by the differences of quantity that how many more people have been saved by $F S$ than by $C S$. It shows the frequencies of event cases sorted by the number of successful evacuees that $F S$ outperforms $C S$. The value in the horizontal axis implies the significance of advantage for $F S$, and the value in the vertical axis indicates in how many cases this level of advantage has been retained. The frequency bars show a distribution that clearly inclines to the right side of the zero point of the horizontal axis.

As can be seen from Figure 1, the largest frequency lies on situations when $F S$ performs equally with $C S$, then the second largest frequency indicates that in 132 event cases $F S$ always saves 10 more people than $C S$. What can also be observed from Figure 1 is that in one case $F S$ saves 36 more people than $C S$, which is the maximum magnitude of advantage exhibited by $F S$ in experiments for these 2040 event cases.

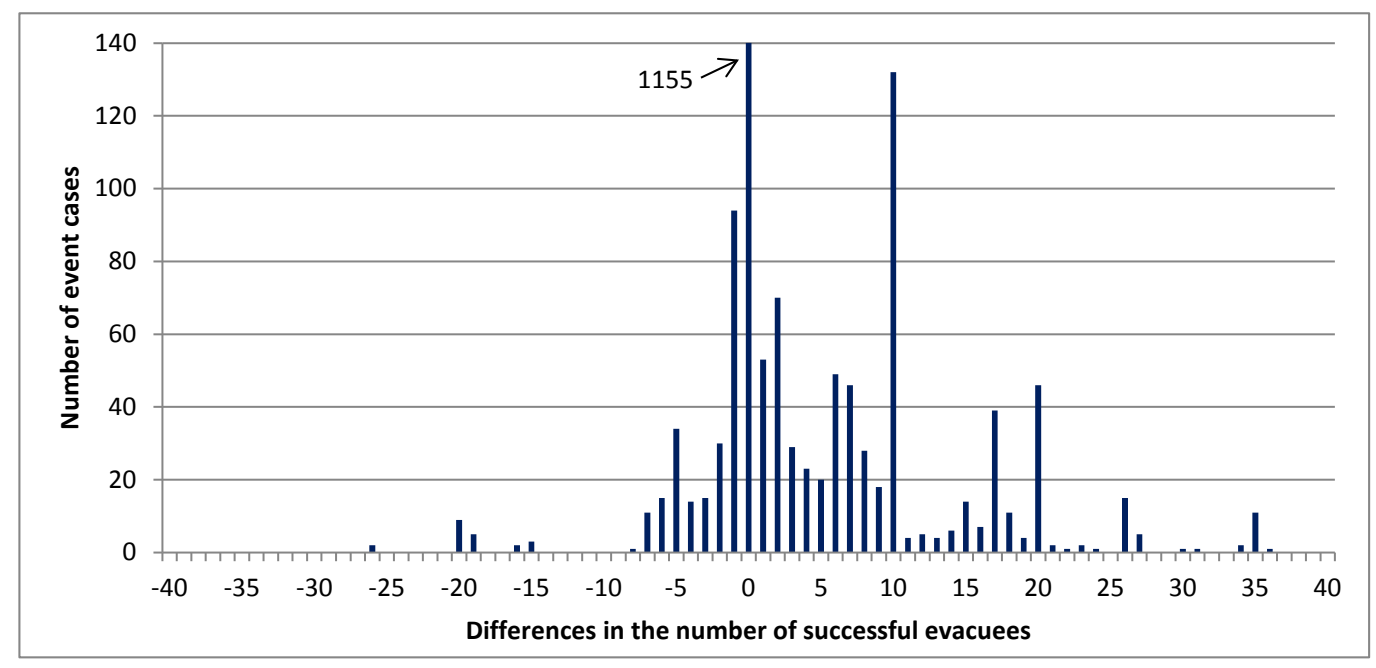

Figure 1. The frequencies of event cases sorted by the difference of successful evacuees between $F S$ and $C S$

Table 2 summarises that, in a large proportion of cases ( $56.6 \%$ and 1155 cases), $F S$ has saved the same number of people as $C S$. The quantity of cases when $F S$ is better is about 3 times the quantity of cases when $C S$ is better. Specifically, in 650 cases (31.9\%), FS outperforms CS, while in 235 cases (11.5\%) CS outperforms FS. FS, when better, saves about 10 people more on average while $C S$, when better, only saves about 4 people more. 
H. Zhao et al., A Fading Memory Model for Indoor Evacuation...

Table 2. Table of comparison between $F S$ and $C S$

\begin{tabular}{|c|c|c|c|}
\hline & Number of cases & Proportion & More saved per case \\
\hline$F S$ is better & 650 & $31.9 \%$ & 9.9 \\
\hline$C S$ is better & 235 & $11.5 \%$ & 4.3 \\
\hline Equal performance & 1155 & $56.6 \%$ & 0 \\
\hline
\end{tabular}

\section{DISCUSSIONS}

For over half of the cases, applying different strategies shows no difference in the number of overall saved people. They may either save all the 150 evacuees, or due to the lack of alternatives a certain number of evacuees are trapped even at the beginning of the events, then different strategies make no significant difference in saving all the other evacuees.

For the cases that the two strategies shows differences, comparisons imply that FS excels $C S$ both in the quantity of better cases (650 and 235 respectively) and in the quality of its advantages as reflected by the average number of more people saved per case (10 and 4 respectively). In more cases fading memory is a better model in terms of evacuation guidance, and decision making can benefit from applying fading memory model trusting more of the knowledge recently explored while devaluing the experience that acquired a long while ago.

There is still a number of cases where fading memory shows worse performance, which throws up fundamental ethical questions: A service devaluing information that actually still is true may come up with suboptimal (evaluation) information. This may raise arguments for whether to adopt a fading memory or not if applying fading memory may not always lead to optimal decision making, and in this cases grasping the out-of-date knowledge would be advantageous.

The heuristics applied in this experiment are arbitrary and may not coincide with the spreading behaviour in fire emergency, which is the main reason why in some cases fading memory deteriorates the results. A sophisticate design of heuristic rules when applying fading memory should improve the performance of fading memory, and ideally in all cases guarantee that applying fading memory excels its counterparts.

\section{CONCLUSIONS}

Spatial knowledge of dynamic environment fades in its value because of its invalidity over time. This paper proposes a fading memory model to represent the knowledge of dynamic environment and presents a firsthand test for the performance of fading memory. Results show that fading memory model is potentially a better representation of the dynamic environment in terms of providing evacuation guidance, which also has implications for all kinds of spatial analysis.

This paper is an attempt and a beginning of exploration for fading memory model applying in dynamic environment decision making. Systematic study will be needed to consolidate this framework with sophisticate heuristics for specific applications. For example, heuristic rules that reflect the realistic mechanism of fire spreading in evacuation can be improved. Besides, the simulation in this paper leaves aside any human behaviour factors such as age and gender, walking speed and visual ability differences of evacuees, congestions caused by evacuee crowds, which may be of benefits if also taken into account when being applied in practice.

\section{REFERENCES}

Afyouni, I., C. Ray, and C. Claramunt (2012, June). Spatial models for context-aware indoor navigation systems: A survey. Journal of Spatial Information Science (4), 85-123.

Ahn, J. and R. Han (2011, December). RescueMe: An indoor mobile augmented-reality evacuation system by personalized pedometry. In 2011 IEEE Asia-Pacific Services Computing Conference (APSCC), pp. 70-77. IEEE.

Amemiya, T., J. Yamashita, K. Hirota, and M. Hirose (2004). Virtual leading blocks for the deaf-blind: A realtime way-finder by verbal-nonverbal hybrid interface and high-density RFID tag space. In Virtual Reality Annual International Symposium, pp. 165-172. 
H. Zhao et al., A Fading Memory Model for Indoor Evacuation...

Becker, T., C. Nagel, and T. H. Kolbe (2009). Supporting contexts for indoor navigation using a multilayered space model. In 2009 Tenth International Conference on Mobile Data Management: Systems, Services and Middleware, pp. 680-685. IEEE.

Fallah, N., I. Apostolopoulos, K. Bekris, and E. Folmer (2013). Indoor human navigation systems: A survey. Interacting with Computers 25(1), 21-33.

Fischer, C., K. Muthukrishnan, M. Hazas, and H. Gellersen (2008). Ultrasound-aided pedestrian dead reckoning for indoor navigation. In Proceedings of the First ACM International Workshop on Mobile Entity Localization and Tracking in GPS-less Environments, New York, New York, USA, pp. 31-36. ACM Press.

Freudenburg, W. (1988). Perceived risk, real risk: social science and the art of probabilistic risk assessment. Science 242(4875), 44-49.

Krek, A. (2002). An agent-based model for quatifying the economic value of geographic information. Ph. D. thesis, Technical University Vienna.

Kulyukin, V., C. Gharpure, J. Nicholson, and G. Osborne (2006, June). Robot-assisted wayfinding for the visually impaired in structured indoor environments. Autonomous Robots 21(1), 29-41.

Lee, J. (2007). A three-dimensional navigable data model to support emergency response in microspatial built-environments. Annals of the Association of American Geographers 97(3), 512-529.

Nguyen, M. H., T. V. Ho, and J. D. Zucker (2013, August). Integration of smoke effect and blind evacuation atrategy (SEBES) within fire evacuation simulation. Simulation Modelling Practice and Theory 36, 44-59.

Park, I., G. U. Jang, S. Park, and J. Lee (2009). Time-dependent optimal routing in micro-scale emergency situation. In 2009 Tenth International Conference on Mobile Data Management: Systems, Services and Middleware, pp. 714-719. IEEE.

Richter, K. F., M. Shi, H. S. Gan, and S. Winter (2013, June). Decentralized evacuation management. Transportation Research Part C: Emerging Technologies 31, 1-17.

Richter, K. F., S. Winter, and S. Santosa (2011). Hierarchical representations of indoor spaces. Environment and Planning B: Planning and Design 38(6), 1052-1070.

Rueppel, U. and K. M. Stuebbe (2008). BIM-based indoor-emergency-navigation-system for complex buildings. Tsinghua Science and Technology 13(Supplement 1), 362-367.

Serrão, M., J. M. F. Rodrigues, J. I. Rodrigues, and J. M. H. Du Buf (2012). Indoor localization and navigation for blind persons using visual landmarks and a GIS. Procedia Computer Science 14, 65-73.

Stahl, C. and T. Schwartz (2010). Modeling and simulating assistive environments in 3-D with the YAMAMOTO toolkit. In 2010 International Conference on Indoor Positioning and Indoor Navigation (IPIN), pp. 1-6.

Tang, F. and A. Ren (2012, March). GIS-based 3D evacuation simulation for indoor fire. Building and Environment 49(1), 193-202.

Wang, J., S. Winter, D. Langerenken, and H. Zhao (2014). Integrating sensing and routing for indoor evacuation. In M. Duckham, E. Pebesma, K. Stewart, and A. Frank (Eds.), Geographic Information Science, Volume 8728 of Lecture Notes in Computer Science, pp. 268-283. Springer International Publishing. 